\title{
Estilo de vida dos universitários: estudo comparativo entre universidade
}

\author{
portuguesa e brasileira.
}

\section{Students' Lifestyle: a comparative study between portuguese and brazilian}

\section{universities.}

\author{
*Rubia Fonseca, *Carvalho, *Amâncio, *Joaquim Escola, *Armando Loureiro. \\ *UTAD- Universidade de Trás os Montes e Alto Douro
}

\begin{abstract}
Resumen
Este estudo apresenta um quadro comparativo entre as semelhanças e diferenças a nível do estilo de vida dos universitários de uma instituição portuguesa e brasileira. Trata-se de um estudo comparativo, do tipo descritivocorrelacional, com recurso a um inquérito por questionário, que inclui uma escala validada, designada por estilo de vida fantástico. Participaram no estudo 1.240 alunos de duas universidades. A maioria dos estudantes $(51,7 \%)$ se enquadrou na categoria geral de um estilo de vida $\underline{\text { Muito }}$ $\underline{\text { Bom. }}$. Em alguns itens da escala do estilo de vida, verificaram-se diferenças significativas entre as duas instituições de ensino superior.

Palavras-chave: Estilo de vida, universitários, educação.
\end{abstract}

\begin{abstract}
This study presents a comparative picture between the similarities and differences in the lifestyle of the university students of a Portuguese and Brazilian institution. This is a comparative, descriptive-correlational study using a questionnaire survey, which includes a validated scale, termed a fantastic lifestyle. 1,240 students from two universities participated in the study. Most students $(51.7 \%)$ were in the general category of a Very Good lifestyle. In some items of the lifestyle scale, there were significant differences between the two institutions of higher education.

Keywords: Lifestyle, university, education.
\end{abstract}

\section{Introdução}

Segundo Zhan (1992) \& Setier (1993) As aspirações por um estilo de vida saudável, consequentemente boa qualidade de vida, são certamente tão antigas quanto à humanidade e têm sido preocupações constantes do ser humano. Para Durkheim (1973) os Estilos de Vida são as "maneiras de agir, pensar e sentir". Neste caso, o sociólogo expande e faz convergir para a definição várias dimensões da pessoa como o sócio-cultural, o psicoafectivo e o biológico-comportamental. De maneira aparentemente mais simples Rocher (1989), definiu E. V. como "as maneiras de viver". Esta aparente simplicidade encerra em si uma complexidade extrema na medida em que faz apelo a um equilíbrio na instabilidade. Isto porque as "maneiras" pressupõem o ajuste de todas as dimensões do sujeito individual à colectividade social e ambiental, e "viver" implica a realização das vontades, desejos e anseios da pessoa.

Sem dúvida, o estilo de vida passou a ser um dos mais importantes determinantes de saúde da população, porém apesar de toda as informações disponíveis sobre o tema, as pesquisas comportamentais revelam que, entre os estudantes universitários, existem cada vez mais comportamentos considerados de risco. (Franca \& Colares, 2008). A OMS demonstra a grande relevância desta pesquisa, ao fazer alusão à importância de se analisar o perfil do estilo de Vida dos Universitários a fim de contribuir para programas eficazes de promoção da saúde. "Um programa eficaz de saúde escolar pode ser um dos mais rentáveis investimentos efetivos que uma nação pode fazer para melhorar simultaneamente a educação e a saúde." OMS (2015) Afinal é demonstrado pela OMS o fato de que "Em todo o mundo, $5 \%$ de todas as mortes de jovens entre as idades de 15 e 29 são atribuíveis ao consumo de álcool”. OMS (2015). É portanto, claramente a faixa etária em que os jovens estão nas Universidades e o momento em que um sistema de prevenção e conscientização e educação em saúde pode ser realizado. A Iniciativa global de saúde escolar da OMS, lançado em 1995, visa mobilizar e fortalecer a promoção da saúde e atividades de educação nos níveis local, nacional, regional e global. A iniciativa destina-se a melhorar a saúde dos alunos, através de escolas. O objetivo da Iniciativa de Saúde Global School da OMS é aumentar o número de escolas que realmente pode ser chamado de "Escolas Promotoras de Saúde". Uma Escola Promotora de Saúde pode ser caracterizada como uma escola que reforça um ambiente saudável para viver, aprender e trabalhar. O próprio conceito proposto pela OMS (1948) compreende a qualidade de vida como sendo a percepção do indivíduo de sua posição na vida, no contexto da cultura e no sistema de valores nos quais ele vive em relação aos seus objetivos, expectativas, padrões e preocupações, e oferece uma perspectiva multidimensional de fatores que estão relacionados entre si. O próprio processo educativo deve conduzir também à saúde do ser humano, inserindo-se também no seu projeto de vida. A educação vê-se, assim, como uma das 
melhores formas para elevar a qualidade e estilo de vida das pessoas (González,1998). O papel do educador como mediador, além de criar condições para o desenvolvimento das competências, é também criar condições para que o homem possa desenvolver as suas capacidades harmônicas.

Nesta linha de pensamento, começamos com uma afirmação de Dias, (1993:4), em que o autor afirma que: " O conceito de educação parece envolver a ideia de um processo de desenvolvimento, de algum modo natural e espontâneo e que se deseja global e harmónico, estruturado e hierarquizado, das capacidades do homem" Ou seja, a educação envolve o desenvolvimento das capacidades do homem. O processo de autoeducação implica, também um processo de construção ao longo da vida com o objetivo de ser mais pleno. Aqui está implícita a procura de um estilo de vida saudável, por parte de todo o ser humano, uma vez que, não há pessoa feliz sem saúde. No dizer de Dias (1982), o homem é o agente da sua própria educação através da interação permanente da sua reflexão e das suas ações. $O$ fator base do melhoramento dos indivíduos, das comunidades e do seu nível de saúde é a educação. No entanto, poderemos interrogarmos acerca do tipo de educação que permite alcançar esses objetivos. Freire (1970), responde a esta questão, ao distinguir dois processos contraditórios de educação: aquele pelo qual os homens caminham no sentido de se tornarem cada vez mais sujeitos, pessoas, tudo e aquele pelo qual são reduzidos a se tornarem cada vez mais objetos, coisas, nada. Uma educação envolvêlo-á no seu projeto pessoal, a outra no projeto de outrem.

Portanto, a percepção de qualidade de vida de um indivíduo passa também pelo seu estilo de vida, pela percepção do seu bem-estar físico, académico, mental, social, psicológico e da sua cosmovisão e qualidade das suas relações. É no âmbito desta problemática que este estudo tem como objetivo geral analisar a relação entre o perfil de competências de estudos, dos estudantes universitários das duas universidades dos dois países (Brasil e Portugal), participantes no estudo.

\section{Metodologia}

Desenvolveu-se um estudo descritivo-correlacional, transversal, de abordagem quantitativa. A amostra foi no total de 1240 alunos, sendo 533 da universidade portuguesa e 707 da universidade brasileira, que frequentavam 10 cursos similares (Biologia, Ciências do Desporto, Comunicação e Multimédia, Educação Básica, Enfermagem, Engenharia Civil, Engenharia Eletrotécnica e de computadores, Engenharia Informática, Línguas, Literatura e Cultura e Psicologia) e que estavam presentes no momento de recolha de dados e aceitaram participar. Estabelecemos como critério de seleção, ser aluno dos cursos selecionados e frequentar o $1^{\circ}, 2^{\circ}$ e $3^{\circ}$ ano dos cursos.

\section{Coleta de dados}

Foi aplicado um questionário de autopreenchimento validado para os dois países. Este instrumento é um questionário com a escala estilos de vida Fantástico (2014).
Antes da aplicação do questionário foi realizado um pré-teste ou teste piloto com uma turma não participante efetivamente do estudo, a fim de que o questionário fosse avaliado e realizado melhorias ou alterações caso necessário, antes da aplicação da versão definitiva. Foi também realizado um pedido de autorização para a realização do estudo à comissão de ética das duas universidades, que deu o seu parecer favorável (7/2016). De seguida foi agendada por email, a recolha de dados com os professores de cada um dos cursos. A investigadora deslocou-se às salas de aula, tendo informado os estudantes e realizada a entrega e recolha dos questionários em sala de aula.

\section{Tratamento de dados}

Para o tratamento dos dados do questionário procedemos à análise de frequências e a análises estatísticas. Para analisar os dados recolhidos utilizou-se o o software estatístico - SPSS (Statistical Package for the Social Sciences), versão 22.0, para Windows. A análise incluiu o uso de estatísticas descritivas (frequências relativas e absolutas, modas, médias e respectivos desvios padrão) e estatística inferencial. Para testar as hipóteses usou-se como referência um nível de significância $=0,05$. Foi construída uma base de dados no SPSS, onde os mesmos foram editados recorrendo-se à estatística descritiva, utilizando as frequências absolutas e relativas, a média e o desvio-padrão da idade e os testes não paramétricos $\chi^{2}$ e Kruskal-Wallis. O nível de significância será de $5 \%$. Como respalda (Fortin \& Filion, 2009) "Na maior parte das investigações, o nível de significância estabelecido é de 0,05.Isto significa que o investigador aceita a probabilidade de se enganar ou de cometer um erro 5 vezes sobre 100. Nos artigos de investigação, os resultados relatados são muitas vezes julgados estatisticamente significativos a menos de 0,05 ou $0,01 . "$

\section{Resultados}

O perfil de Estilo de Vida abrangeu as variáveis Escala estilo de vida Fantástico (2014), família e amigos, atividade física, nutrição, cigarro e drogas, álcool, sono, cinto de segurança, estresse e sexo seguro, tipo de comportamento, introspecção e trabalho.

No total da amostra $(n=1240)$, conforme podemos visualizar na tabela 1, a maioria dos estudantes se enquadrou na categoria geral de um estilo de vida Muito $\underline{B o m}$, que segundo a escala a pontuação vai de (70 a 84), indicando que o estilo de vida proporciona adequada influência para a saúde $(51,7 \%)$. Na análise das categorias específicas, começando por família e amigos, $(57,4 \%)$ está excelente, $(28,3 \%)$ está na categoria de uma boa atividade física, $(43,6 \%)$ tem sua nutrição muito boa, sobre o uso de cigarro e drogas $(91,8 \%)$ está excelente, no consumo de álcool $(94,0 \%)$ se encontra na categoria excelente, $(32,1 \%)$ tem um sono bom, $(68,7 \%)$ está na categoria excelente quanto ao uso do cinto de segurança, $(39,1 \%)$ recebeu muito bom, na questão do estress, sobre o sexo seguro $(61,7 \%)$ está excelente, sobre o tipo de comportamento $(40,2 \%)$ se enquadra no muito bom, na introspecção $(41,1 \%)$ está no muito bom e quanto a satisfação no trabalho/curso universitário $(31,5 \%)$ está na 
categoria muito bom. Na ocupação dos tempos livres, (54,8\%) afirma assistir Tv/ouvir músicas, (4,5\%) afirmam ir ao teatro, $(22,6 \%)$ vão ao cinema, $(45,3 \%)$ assistem séries em casa, (15,5\%) afirmam assistir competições desportivas, $(33,4 \%)$ saem para dançar/frequentar cafés/discotecas/encontrar amigos, (4,5\%) vão a shows, (22,4\%) jogam baralho, bingo/videogame, $(64,0 \%)$ ficam navengando na internet em seus tempos livres e $(28,5 \%)$ assistem filmes em casa e $(5,9 \%)$ vão a concertos. Sobre as redes sociais mais utilizadas $(66,1 \%)$ utilizam mais o facebook, o Instagram fica em segundo lugar com $(15,6 \%)$ e o Snapchat em terceiro lugar com $(8,9 \%)$. Os gêneros de filmes mais apreciados são comédia com $(41,1 \%)$ e aventura/ação $(31,6 \%)$.

$\mathrm{Na}$ amostra dos estudantes Portugueses $(n=533)$, a maioria dos estudantes se enquadrou na categoria geral de um estilo de vida Muito Bom, indicando que o estilo de vida proporciona adequada influência para a saúde $(38,5 \%)$. $\mathrm{Na}$ análise das categorias específicas, começando por família e amigos, $(59,3 \%)$ está excelente, $(29,5 \%)$ está na categoria de uma boa atividade física, $(43,5 \%)$ tem sua nutrição muito boa, sobre o uso de cigarro e drogas $(78,0 \%)$ está excelente, no consumo de álcool $(82,9 \%)$ se encontra na categoria excelente, $(28,9 \%)$ tem um sono muito bom, $(74,9 \%)$ está na categoria excelente quanto ao uso do cinto de segurança, (37,3\%) está excelente na questão do estress, sobre o sexo seguro $(62,7 \%)$ está excelente, sobre o tipo de comportamento $(45,2 \%)$ se enquadra no muito bom, na introspecção 38,6 está no muito bom e no trabalho/curso universitário $(38,6 \%)$ está na categoria muito bom.

$\mathrm{Na}$ amostra dos estudantes Brasileiros $(n=707)$, a maioria dos estudantes se enquadrou na categoria geral de um estilo de vida Muito Bom, indicando que o estilo de vida proporciona adequada influência para a saúde $(50,8 \%)$. Na análise das categorias específicas, começando por família e amigos, $(55,3 \%)$ está excelente, $(28,2 \%)$ está na categoria de uma atividade física regular, $(39,4 \%)$ tem sua nutrição muito boa, sobre o uso de cigarro e drogas $(97,2 \%)$ está excelente, no consumo de álcool $(97,2 \%)$ se encontra na categoria excelente, $(31,1 \%)$ tem um sono bom, $(62,9 \%)$ está na categoria excelente quanto ao uso do cinto de segurança, (40,2\%) está muito bom na questão do estress, sobre o sexo seguro $(57,4 \%)$ está excelente, sobre o tipo de comportamento $(35,6 \%)$ se enquadra no muito bom, na introspecção $(42,0 \%)$ está no muito bom e no trabalho/curso universitário $(29,1 \%)$ está na categoria excelente.
Tabela 1.

Perfil de estilo de vida dos estudantes por universidades e total

\begin{tabular}{|c|c|c|c|}
\hline Variáveis/Categorias & Brasil & Portugal & $\begin{array}{c}\text { Total } \\
(\%)\end{array}$ \\
\hline \multicolumn{4}{|l|}{ Categorias: } \\
\hline $\begin{array}{l}\text { Estilo de Vida Total } \\
\text { Necessita melhorar } \\
\text { Regular } \\
\text { Bom } \\
\text { Muito Bom } \\
\text { Excelente } \\
\end{array}$ & $\begin{array}{l}0,3 \\
5,8 \\
27,9 \\
\mathbf{5 0 , 8} \\
15,3 \\
\end{array}$ & $\begin{array}{l}0 \\
2,8 \\
34,6 \\
\mathbf{5 2 , 6} \\
10,0 \\
\end{array}$ & $\begin{array}{l}0,1 \\
4,3 \\
31,3 \\
\mathbf{5 1 , 7} \\
12,6\end{array}$ \\
\hline $\begin{array}{l}\text { Família e amigos } \\
\text { Necessita melhorar } \\
\text { Regular } \\
\text { Bom } \\
\text { Muito Bom } \\
\text { Excelente }\end{array}$ & $\begin{array}{l}0,6 \\
2,2 \\
13,0 \\
28,8 \\
\mathbf{5 5 , 3}\end{array}$ & $\begin{array}{l}0,9 \\
1,5 \\
11,0 \\
26,6 \\
\mathbf{6 0 , 0}\end{array}$ & $\begin{array}{l}0,8 \\
1,9 \\
12,1 \\
27,8 \\
\mathbf{5 7 , 4}\end{array}$ \\
\hline $\begin{array}{l}\text { Atividade Física } \\
\text { Necessita melhorar } \\
\text { Regular } \\
\text { Bom } \\
\text { Muito Bom } \\
\text { Excelente } \\
\end{array}$ & $\begin{array}{l}18,9 \\
\mathbf{2 8 , 2} \\
26,9 \\
16,0 \\
9,9 \\
\end{array}$ & $\begin{array}{l}12,8 \\
27,3 \\
\mathbf{3 0 , 0} \\
16,3 \\
13,6 \\
\end{array}$ & $\begin{array}{l}16,2 \\
27,8 \\
\mathbf{2 8 , 3} \\
16,1 \\
11,5 \\
\end{array}$ \\
\hline $\begin{array}{l}\text { Nutrição } \\
\text { Necessita melhorar } \\
\text { Regular } \\
\text { Bom } \\
\text { Muito Bom } \\
\text { Excelente } \\
\end{array}$ & $\begin{array}{l}1,2 \\
8,3 \\
29,4 \\
\mathbf{3 9 , 4} \\
21,7 \\
\end{array}$ & $\begin{array}{l}1,0 \\
3,4 \\
28,1 \\
\mathbf{4 8 , 6} \\
18,9 \\
\end{array}$ & $\begin{array}{l}1,1 \\
6,0 \\
28,8 \\
\mathbf{4 3 , 6} \\
20,4\end{array}$ \\
\hline $\begin{array}{l}\text { Cigarro e drogas } \\
\text { Necessita melhorar } \\
\text { Regular } \\
\text { Bom } \\
\text { Muito Bom } \\
\text { Excelente } \\
\end{array}$ & $\begin{array}{l}0 \\
0,2 \\
1,1 \\
1,5 \\
\mathbf{9 7 , 2} \\
\end{array}$ & $\begin{array}{l}0 \\
0,6 \\
3,7 \\
9,7 \\
\mathbf{8 5 , 9} \\
\end{array}$ & $\begin{array}{l}0 \\
0,4 \\
2,4 \\
5,4 \\
\mathbf{9 1 , 8}\end{array}$ \\
\hline $\begin{array}{l}\text { Álcool } \\
\text { Necessita melhorar } \\
\text { Regular } \\
\text { Bom } \\
\text { Muito Bom } \\
\text { Excelente } \\
\end{array}$ & $\begin{array}{l}0 \\
0,2 \\
0,4 \\
2,3 \\
\mathbf{9 7 , 1} \\
\end{array}$ & $\begin{array}{l}0,4 \\
0,8 \\
2,5 \\
5,9 \\
\mathbf{9 0 , 4} \\
\end{array}$ & $\begin{array}{l}0,2 \\
0,5 \\
1,3 \\
4,0 \\
\mathbf{9 4 , 0}\end{array}$ \\
\hline $\begin{array}{l}\text { Sono } \\
\text { Necessita melhorar } \\
\text { Regular } \\
\text { Bom } \\
\text { Muito Bom } \\
\text { Excelente } \\
\end{array}$ & $\begin{array}{l}5,1 \\
12,5 \\
\mathbf{3 1 , 1} \\
26,9 \\
24,4 \\
\end{array}$ & $\begin{array}{l}2,5 \\
8,2 \\
\mathbf{3 3 , 4} \\
29,2 \\
26,8 \\
\end{array}$ & $\begin{array}{l}3,9 \\
10,6 \\
\mathbf{3 2 , 1} \\
27,9 \\
25,4 \\
\end{array}$ \\
\hline $\begin{array}{l}\text { Cinto de segurança } \\
\text { Necessita melhorar } \\
\text { Regular } \\
\text { Bom } \\
\text { Muito Bom } \\
\text { Excelente }\end{array}$ & $\begin{array}{l}1,2 \\
2,3 \\
10,7 \\
22,9 \\
\mathbf{6 2 , 9}\end{array}$ & $\begin{array}{l}1,1 \\
2,1 \\
7,6 \\
13,1 \\
\mathbf{7 6 , 0}\end{array}$ & $\begin{array}{l}1,2 \\
2,2 \\
9,3 \\
18,6 \\
\mathbf{6 8 , 7}\end{array}$ \\
\hline $\begin{array}{l}\text { Estresse } \\
\text { Necessita melhorar } \\
\text { Regular } \\
\text { Bom } \\
\text { Muito Bom } \\
\text { Excelente } \\
\end{array}$ & $\begin{array}{l}0,2 \\
4,4 \\
24,9 \\
\mathbf{4 0 , 2} \\
30,4 \\
\end{array}$ & $\begin{array}{l}0,4 \\
2,3 \\
21,6 \\
37,8 \\
\mathbf{3 8 , 0} \\
\end{array}$ & $\begin{array}{l}0,3 \\
3,5 \\
23,4 \\
\mathbf{3 9 , 1} \\
33,7 \\
\end{array}$ \\
\hline $\begin{array}{l}\text { Sexo seguro } \\
\text { Necessita melhorar } \\
\text { Regular } \\
\text { Bom } \\
\text { Muito Bom } \\
\text { Excelente }\end{array}$ & $\begin{array}{l}14,4 \\
3,2 \\
11,4 \\
13,5 \\
\mathbf{5 7 , 4}\end{array}$ & $\begin{array}{l}4,4 \\
4,2 \\
10,4 \\
14,5 \\
\mathbf{6 6 , 5}\end{array}$ & $\begin{array}{l}9,7 \\
3,7 \\
10,9 \\
14,0 \\
\mathbf{6 1 , 7} \\
\end{array}$ \\
\hline
\end{tabular}




\begin{tabular}{l|l|l|l}
\hline Tipo de comportamento & & & \\
Necessita melhorar & 2,1 & 1,1 & 1,7 \\
Regular & 9,3 & 5,3 & 7,6 \\
Bom & 34,6 & 26,9 & 31,2 \\
Muito Bom & $\mathbf{3 5 , 6}$ & $\mathbf{4 6 , 0}$ & $\mathbf{4 0 , 2}$ \\
Excelente & 18,3 & 20,6 & 19,3 \\
\hline Introspeçaão & & & \\
Necessita melhorar & 0,5 & 0,6 & 0,5 \\
Regular & 5,5 & 2,5 & 4,2 \\
Bom & 25,2 & 30,1 & 27,3 \\
Muito Bom & $\mathbf{4 2 , 0}$ & $\mathbf{4 0 , 0}$ & $\mathbf{4 1 , 1}$ \\
Excelente & 27,0 & 26,8 & 26,9 \\
\hline Trabalho & & & \\
Necessita melhorar & 5,0 & 1,5 & 3,4 \\
Regular & 8,9 & 7,4 & 8,2 \\
Bom & 28,7 & 33,4 & 30,8 \\
Muito Bom & 28,4 & $\mathbf{3 5 , 5}$ & $\mathbf{3 1 , 5}$ \\
Excelente & $\mathbf{2 9 , 1}$ & 22,2 & 26,1 \\
\hline
\end{tabular}

Para testar a hipótese: Existe diferença entre o perfil de estilo de vida (Escala estilo de vida Fantástico (2014), família e amigos, atividade física, nutrição, cigarro e drogas, álcool, sono, cinto de segurança, estresse e sexo seguro, tipo de comportamento, introspecção e trabalho, dos estudantes entre as universidades dos dois países, procedemos à realização dos testes estatísticos de $\chi^{2} \mathrm{e}$ Kruskal-Wallis (KW). KW: $\mathrm{P}=0,120$ portanto, em relação as categorias do estilo de vida e as universidades, as diferenças são altamente significativas Kw: $\mathrm{P} \leq 0,000$.

A universidade brasileira 395,48 , tem uma média de ordenação maior no estilo de vida do que a universidade portuguesa 375,78 . Portanto, verificaram-se diferenças significativas no total da escala do estilo de vida, $\chi^{2}: \mathrm{p} \leq$ 0,017 ,onde há $+2,2$ casos que o esperado na categoria excelente na universidade brasileira, sendo $15,3 \%$ versus $10,0 \%$ de alunos da universidade portuguesa.

Na categoria Atividade Física T-studant: $\mathrm{p} \leq 0,000$ as diferenças são altamente significativas. $\mathrm{Na}$ universidade portuguesa 3,43 tem uma média de frequência maior de atividade física do que a universidade brasileira.

Na Nutrição , $\chi^{2}: p \leq 0,002$, onde na categoria regular na nutrição há $+3,3$ casos de alunos que o esperado na universidade brasileira. Dos alunos que se enquadram no regular na nutrição $8,3 \%$ são da universidade brasileira e $3,4 \%$ são da universidade portuguesa. Na categoria muito bom, foram encontrados $+3,0$ casos que o esperado e $48,6 \%$ são da universidade portuguesa e $39,4 \%$ são da universidade brasileira. A atividade física e os hábitos alimentares são dois elementos do estilo de vida na parte física que desempenham um papel significativo na promoção da saúde e na prevenção de diversas doenças. (Silva et al, 2011).

Sobre as Drogas e Tabaco $\chi^{2:} \mathrm{p} \leq 0,000$ verificaram-se diferenças altamente significativas. $\mathrm{Na}$ categoria Excelente $+6,6$ casos que o esperado na universidade brasileira com $85,9 \%$ de alunos da universidade portuguesa e $97,2 \%$ de alunos na universidade brasileira. Pedimos a simulação de Monte Carlo e o resultado foi o mesmo $\chi^{2}: \mathrm{p} \leq 0,000$. O tabagismo é uma doença crónica que acusa dependência física e psíquica, sendo que considerada pela WHO (2003), como sendo a principal causa evitável de morbilidade e mortalidade do mundo. No Consumo de álcool segundo os dados obtidos verificaram-se diferenças altamente significativas $\chi^{2}: \mathrm{p} \leq$ 0,000, Na categoria Excelente $+4,6$ casos que o esperado na universidade brasileira, sendo $90,4 \%$ na universidade portuguesa versus $97,1 \%$ da universidade brasileira. Pedimos a simulação de Monte Carlo e o resultado permaneceu sendo $\chi^{2}: \mathrm{p} \leq 0,000$. Também pode-se dizer que após a entrada no mundo acadêmico, muitas vezes consolidam-se ou iniciam-se comportamentos menos saudáveis, nomeadamente o tabagismo ou o aumento do consumo de bebidas alcoólicas. (Gracio,2009 \& Parkerson,1990) dentro destas práticas não saudáveis na dimensão física, estudos concluíram que vem sendo cada vez mais observado na população de graduandos o aumento do consumo, principalmente de substâncias ilícitas e de bebidas alcoólicas.(Ramos-Dias et al, 2010 \& Kerr-Corrêa,1999).

Sobre a questão do Sono $\chi^{2}: \mathrm{p} \leq 0,017$. Na subcategoria Necessita melhorar, $+2,3$ casos que o esperado na universidade brasileira, sendo $2,5 \%$ dos alunos da universidade portuguesa versus $5,1 \%$ da universidade brasileira. $\mathrm{Na}$ subcategoria regular $+2,4$ casos que $\mathrm{o}$ esperado na universidade brasileira sendo $8,2 \%$ de alunos da universidade portuguesa versus $12,5 \%$ de alunos da universidade brasileira. Uma boa noite de sono, desempenha um papel fundamental no bem-estar físico, psíquico e social dos indivíduos, é também benéfica para o cérebro, exerce influência no bom funcionamento da memória e na capacidade de absorção de informação portanto, dormir bem é essencial para quem estuda. (Ancoli-Israel et al., 2011). Sobre o uso do Cinto de segurança $\chi^{2}: p \leq 0,000$. Na subcategoria excelente $+4,8$ casos que o esperado na universidade portuguesa, sendo $76,0 \%$ de alunos da universidade portuguesa versus $62,9 \%$ de alunos da universidade brasileira. Sobre o Stress $\chi^{2}: p \leq 0,025$, Na subcategoria regular, $+2,0$ casos que o esperado na universidade brasileira, sendo $2,3 \%$ de alunos da universidade portuguesa versus $4,4 \%$ de alunos da universidade brasileira. $\mathrm{Na}$ subcategoria excelente + 2,8 casos que o esperado na universidade portuguesa, sendo $38 \%$ de alunos da universidade portuguesa versus $30,4 \%$ de alunos da universidade portuguesa. Sobre a questão do sexo seguro $\chi^{2}: p \leq 0,000$. Na subcategoria necessita melhorar $+5,5$ casos que o esperado na universidade brasileira, sendo $4,4 \%$ de alunos da universidade portuguesa versus $14,4 \%$ de alunos da universidade portuguesa. Na subcategoria excelente $+3,1$ casos que o esperado de alunos da universidade portuguesa, sendo $66,5 \%$ de alunos da universidade portuguesa versus $57,4 \%$ de alunos da universidade brasileira. De acordo com Spizzichino et al(2005), as infecções entre os jovens constituem uma importante parcela das novas infecções por HIV na população mundial, sendo este segmento populacional considerado uma das populações-alvo para ações de educação e prevenção. Comportamentos de introspeç̧ão $\chi^{2}: \mathrm{p} \leq$ 0,000 . Na subcategoria regular $+2,6$ casos que o esperado na universidade brasileira, sendo $5,3 \%$ de alunos da universidade portuguesa versus $9,3 \%$ de alunos da universidade brasileira. $\mathrm{Na}$ subcategoria muito bom $+3,6$ casos que o esperado na universidade portuguesa, sendo $46 \%$ de alunos da universidade portuguesa versus $35,6 \%$ 
de alunos da universidade brasileira. Sobre a satisfação no trabalho/estudos, $\chi^{2}: \mathrm{p} \leq 0,000$. Na subcategoria Necessita melhorar $+3,2$ casos que o esperado na universidade brasileira, sendo $1,5 \%$ de alunos da universidade portuguesa versus $5,0 \%$ de alunos da universidade brasileira. $\mathrm{Na}$ subcategoria excelente $+2,7$ casos que o esperado na universidade brasileira.

Esta curiosidade em pesquisar sobre o estilo de vida em nossas amostras, nos remete ao fato de que nos dias de hoje, quando se fala em sucesso académico, não se restringe a sua operacionalização ao êxito ou aprovação nas unidades curriculares. É necessário contemplar-se, igualmente, o desenvolvimento do bem-estar psicossocial dos estudantes (Castro \& Almeida, 2016). Apesar de serem considerados uma população privilegiada, os problemas psicológicos em jovens universitários são tão prevalentes como nos jovens da mesma idade que não frequentam a universidade. Estes problemas têm vindo a aumentar em número e gravidade nos últimos anos a nível internacional, com cerca de 12 a $18 \%$ dos estudantes universitários com diagnóstico de problemas psicológicos (Hunt \& Eisenberg, 2010; Mowbray, Mandiberg, Stein, Hopels, Curlin, Megivern \& Lett, 2006), tais como o uso excessivo de álcool (Blanco, Okuda, Wright, Hasin, Grant, Liu \& Olfson, 2008) e drogas (Kitzrow, 2003) ameaças à autoestima, ansiedade e depressão, stresse, suicídio, problemas alimentares e de atividade sexual (East, Peters, O'Brien, \& Jackson, 2010).

\section{Conclusão}

De um modo geral, conclui-se que os estudantes das duas universidades apresentam um estilo de vida muito bom, indicando que o estilo de vida proporciona adequada influência para a saúde. Os resultados obtidos permitiram identificar itens da escala do estilo de vida que devem ser melhor desenvolvidas, com vista à adoção de medidas que promovam melhor estilo de vida contínuo de cada universitário para o sucesso académico.

\section{Referencias}

Ancoli-Israel, S., Bliwise, D. L. e Norgoard, J.P. (2011). The effect of nocturia on sleep. Sleep Medicine Reviews, 15 (2), pp. 91-97.

Anez,C.R.,Reis,R.R., \& Petroski, E.L (2008). Versão brasileira do questionário "Estilo de Vida Fantástico". Tradução e validação para adultos jovens. ArqBras Cardiol ;91(2):102-109.

Blanco, C., Okuda, M., Wright, C., Hasin, D.S., Grant, B. F., Liu, S.M., \& Olfson, M.(2008). Mental health of college students and their non-college Attending peers: Results from the National Epidemiologic Study on Alcohol and Related Conditions. Archives of General Psychiatry, 65.

Dias,J.R.(1993) "Filosofia da Educação. Pressupostos, funções, método, estatuto". Revista Portuguesa de Filosofia.

Durkhei, Émile. (1973). As regras do método sociológico. São Paulo. Abril Cultural, v. 33 (Coleção Os Pensadores).
East, L., Peters, K., O’Brien, L., \& Jackson, D. (2010). Disrupted sense of self: Young women and sexually transmitted infections, Journal of Clinical Nursing, 19.

Franca C, Colares V.(2008). Estudo comparativo de condutas de saúde entre universitários no início e no final do curso. Rev. Saúde Pública.

Freire, P. (1970). Pedagogia do oprimido. Rio de Janeiro: Editora Paz e Terra.

Fortin, M.F; Coté.J; Filion.F. (2009). Fundamentos e etapas do processo de investigação. Loures: Lusodidática.

Gonzalez,M.I.S (1998). La educación para la salud del siglo XXI. Comunicación y salud. Madrid: Diaz de Santos.

Grácio JCG.(2009). Determinantes do consumo de bebidas alcoólicas nos estudantes do ensino superior de Coimbra. [Dissertação de Mestrado]. Coimbra: Universidade de Coimbra.

Hunt, J., \& Eisenberg, D (2010). Mental Health problems and help-seeking behavior among college students. Journal of Adolescent Health.

Kerr-Corrêa F, Andrade AG, Bassit AZ, Boccuto NMVF.(1999). Uso de álcool e drogas por estudantes de medicina da Unesp. Rev Bras Psiquiatr. 21(2):95100 .

Kitzrow, M.A. (2003). The mental health needs of today's college students: Challenges and recommendations. Journal of Student Affairs Research and Practice.

Mowbray, C.T., Stein, C.H., Kopels, S., Curlin, C., Megivern, Lett, R. (2006).Campus mental health services: Recommendations for change. American Journal of Orthopsychiatry.

OMS. Organizacion Mundial de La Salud. (2015). Documentos Básicos $47^{\mathrm{a}}$ Ed.Genebra.

Parkerson GR Jr, Broadhead WE, Tse CK.(1990). The health status and life satisfaction of first-year medical students. Acad Med. 65(9):586-8.

Ramos-Dias JC, Libardi MC, Zillo CM, Igarashi MH, Senger MH.(2010). Qualidade de vida em cem alunos do curso de Medicina de Sorocaba-PUC/SP. Rev Bras Educ Med. 34(1):116-23.

Rocher, G. (1989) Sociologia Geral. Acção social. Lisboa: Editorial Presença.

Setiér Santamaría ML.(1993). Indicadores sociales de qualidad de vida: um sistema de medición aplicado al pais. Madrid: Centro de Investigaciones Sociológicas.

Silva D.A, Quadros TM, Gordia AP, Petroski EL.(2011). Associação do sobrepeso com variáveis sóciodemográficas e estilo de vida em universitários. Cienc Saude Colet. 16(11):4473-9.

Spizzichino L, Piccinno F, Pedone G, Gallo P, Valli R, Cotti L, Bianconi M, Luzi AM.(2005). Adolescenti e HIV: Le champagne informat ive dei giovani per i Giovanni. Ann Ist Super Sanita. 41(1):113-8.

UNESCO.(2011).Global Education Digest.Paris: Institute for Statistics.

WHO.World Health Organization(2005). Alcohol policy in the WHO European Region: current status and the way forward. Copenhagen: Regional Office for Europe. 
FONSECA, ESCOLA, CARVALHO, LOUREIRO

Zhan L. (1992). Quality of life: conceptual and measurement issues. J AdvNurs; 17 (7). 